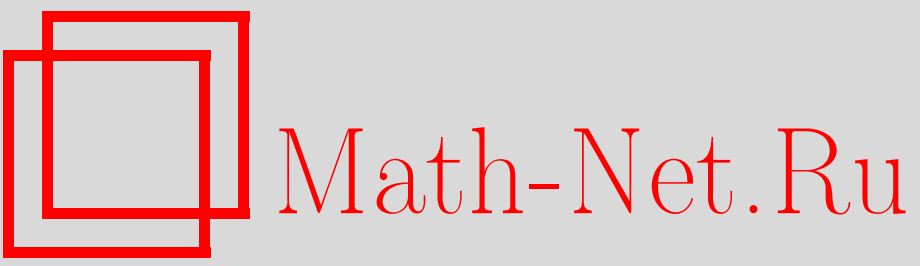

А. Л. Якымив, Предельная теорема для средних членов вариационного ряда длин циклов случайной $A$-подстановки, Теория вероятн. и ее примен., 2009, том 54, выпуск 1, 63-79

DOI: https://doi.org/10.4213/tvp2499

Использование Общероссийского математического портала Math-Net.Ru подразумевает, что вы прочитали и согласны с пользовательским соглашением

http://www.mathnet.ru/rus/agreement

Параметры загрузки:

IP : 54.92.164.108

26 апреля 2023 г., 09:58:20 


\title{
ПРЕДЕЛЬНАЯ ТЕОРЕМА ДЛЯ СРЕДНИХ ЧЛЕНОВ ВАРИАЦИОННОГО РЯДА ДЛИН ЦИКЛОВ СЛУЧАЙНОЙ $А$-ПОДСТАНОВКИ ${ }^{1)}$
}

\begin{abstract}
Рассматривается случайная подстановка $\tau_{n}$, равномерно распределенная на множестве всех подстановок степени $n$, длины циклов которых принадлежат фиксированному множеству $A$ (так называемых $A$-подстановок). Пусть $\zeta_{n}$ - общее число циклов и $\eta_{n}(1) \leqslant \eta_{n}(2) \leqslant \cdots \leqslant \eta_{n}\left(\zeta_{n}\right)$ - вариационный ряд длин циклов подстановки $\tau_{n}$. В настоящей статье получена центральная предельная теорема для средних членов этого ряда, а именно для случайных величин $\eta_{n}(m)$ с номерами $m=\alpha \ln n+o(\sqrt{\ln n})$ при $n \rightarrow \infty$ и фиксированном $\alpha \in(0, \sigma)$ для определенного класса множеств $A$ положительной асимптотической плотности $\sigma$. Основным средством доказательства является новая трехмерная тауберова теорема. Поведение крайних левых и крайних правых членов этого ряда исследовано автором ранее.
\end{abstract}

Ключевые слова и фразы: случайная $A$-подстановка, вариационный ряд длин циклов подстановки, тауберова теорема.

1. Введение. Пусть $A-$ произвольное непустое подмножество множества натуральных чисел N. Подстановки, длины циклов которых принадлежат множеству $A$, называются $A$-подстановками (см. [20]). Пусть $T_{n}=T_{n}(A)$ есть множество $A$-подстановок степени $n$ и $\tau_{n}-$ случайная подстановка, равномерно распределснная на $T_{n}$. Через $\zeta_{m n}$ обозначим число циклов подстановки $\tau_{n}$, имеющих длину $m \in \mathbf{N}$. Ясно, что $\zeta_{m n}=0$ при $m \notin A$. Далее, пусть $\zeta_{n}$ - общее число циклов случайной подстановки $\tau_{n}$, т.е.

$$
\zeta_{n}=\sum_{m \in \mathbf{N}} \zeta_{m n}
$$

Случайные $A$-подстановки являются объектом пристального внимания ряда авторов на протяжении последних тридцати с лишним лет. Обзор по этой тематике можно найти в статье автора [22] и книге [23]. Одними

\footnotetext{
* Математический институт им. В. А. Стеклова РАН, ул. Губкина, 8, 119991 Москва, Россия; e-mail: arsen@mi.ras.ru

1) Работа выполнена при поддержке Российского фонда фундаментальных исследований (гранты № 08-01-00563, 09-01-00724).
} 
из первых работ в этом направлении были статьи [1], [2], [11], [12]. Еще раньше в работах [17], [18] были рассмотрены более общие объекты случайные отображения с ограничениями на длины циклов. При этом для тех или иных классов множеств $A$ изучались задачи асимптотического поведения при $n \rightarrow \infty$ числа элементов множества $T_{n}(A)$ и предельного поведения (в слабом смысле) случайных величин $\zeta_{m n}$ и $\zeta_{n}$, где $m$ - фиксированный элемент множества $A$. Имеется пять монографий, содержащих сведения об $A$-подстановках: [7], [10], [19], [20], [23].

Расположим длины циклов случайной подстановки $\tau_{n}$ в возрастающем порядке:

$$
\eta_{n}(1) \leqslant \eta_{n}(2) \leqslant \cdots \leqslant \eta_{n}\left(\zeta_{n}\right) .
$$

Через $|X|$ мы будем везде далее обозначать число элементов конечного множества $X$. В настоящей статье мы будем предполагать, что

$$
p(n) \equiv \frac{\left|T_{n}(A)\right|}{n !}=n^{\sigma-1} L(n), \quad n \in \mathbf{N},
$$

где функция $L(n)$ - медленно меняется на бесконечности (см. [21]), а $\sigma \in(0,1]$. Имеется широкий класс примеров множеств $A$, для которых выполнено (2) (см. [1], [3], [8], [9], [13]-[16], [23, гл. 3]), более подробный обзор этих работ содержится в [22].

Экстремальные свойства случайных подстановок, случайных отображений и других комбинаторных структур явились предметом изучения со стороны целого ряда авторов, начиная с известной работы [4] (история вопроса и подробные ссылки имеются в работе [5]). Отметим также, что обзор работ в этом направлении до 1984 г. содержится в монографии [7]. В частности, исследовано и предельное поведение случайных величин $\eta_{n}(m)$ при $n \rightarrow \infty$ и $m=\alpha \ln n+o(\sqrt{\ln n})$ с $\alpha \in(0,1)$, когда $A=\mathbf{N}$ (см. [7, раздел 1.9 , теорема 4$]$ ).

Что же касается случайных $A$-подстановок, то здесь пока изучено только поведение крайних правых и крайних левых членов вариационного ряда (1) (см. статьи [22], [25]). В настоящей работе получена предельная теорема для случайных величин $\eta_{n}(m)$, находящихся в средней части вариационного ряда (1), при $n \rightarrow \infty$ и различном характере поведения $m$.

Доказательство этой предельной теоремы для $\eta_{n}(m)$ опирается на новую трехмерную тауберову теорему (п. 2, теорема 3 ), которая обобщает соответствующий результат из книги $[23$, раздел 1.5 , теорема 1.5.7]. Отметим, что тауберовы теоремы нашли широкое применение в теории вероятностей. Это относится, в частности, к таким областям, как теория ветвящихся процессов, теория восстановления, теория рекордов, теория безгранично делимых распределений, теория суммирования независимых случайных величин, комбинаторная теория вероят- 
ностей и др. За более подробной информацией по этому поводу читатель может обратиться к недавно вышедшей книге [23].

2. Основной результат. Далее будет доказана следующая теорема.

Теорема 1. Пусть выполнено соотношение (2) и

$$
l(n) \equiv \sum_{i \in A(n)} \frac{1}{i}=\sigma \ln n+o(\sqrt{\ln n}) \quad(n \rightarrow \infty),
$$

где $A(n)=A \cap[1, n]$, и пусть положительная последовательность $g(n) \rightarrow \infty$ такова, что

$$
\frac{\sigma \ln n-g(n)}{\sqrt{\ln n}} \longrightarrow+\infty \quad(n \rightarrow \infty)
$$

Тогда при $n \rightarrow \infty$ и $m=g(n)+o(\sqrt{g(n)})$ последовательность случайных величин

$$
\frac{\sigma \ln \eta_{n}(m)-m}{\sqrt{m}}
$$

слабо сходится $к$ стандартному нормальному закону.

Отметим, что в предположениях теоремы 1

$$
\frac{\zeta_{n}-\sigma \ln n}{\sqrt{\sigma \ln n}}
$$

слабо сходится при $n \rightarrow \infty$ к стандартному нормальному закону [24], так что случайные величины $\eta_{n}(m)$ с указанными номерами $m$ действительно находятся в средней части вариационного ряда (1).

П р и м е р ы. 1 . Пусть $g(n)=\alpha \ln n$, где $\alpha \in(0, \sigma)$. Тогда для $g(n)$ справедлива теорема 1 (при $A=N$ соответствующий пример разобран в книге [7, раздел 1.9 , теорема 4 , раздел 1.10 , теорема 3$])$.

2. Пусть $g(n) \rightarrow \infty, g(n)=o(\ln n)$ при $n \rightarrow \infty$. Тогда для $g(n)$ справедлива теорема 1 . Отметим, что в этом случае номера $m=g(n)+$ $o(\sqrt{g(n)})$ отличаются от тех, которые ранее принято было называть средними (см. ссылку в примере 1 ).

Отметим, что следствием (2) является соотношение

$$
l(n) \equiv \sum_{i \in A(n)} \frac{1}{i}=\sigma \ln n+o(\ln n) \quad(n \rightarrow \infty)
$$

(см. [15]), т.е. в соотношении (3) требуется несколько большее, чем (4). Положим при $k, n \in \mathbf{N}$

$$
\zeta_{n}(k)=\sum_{i \leqslant k} \zeta_{\text {in }}
$$


$\zeta_{0}(k)=\zeta_{n}(0)=\zeta_{0}(0)=0$. В основе доказательства теоремы 1 лежит известное равенство

$$
\mathbf{P}\left\{\eta_{n}(m) \leqslant r\right\}=\mathbf{P}\left\{\zeta_{n}(r) \geqslant m\right\},
$$

справедливое для произвольных натуральных $r, m \leqslant n$ (см. [7, раздел 1.10, равенство (9)]), и следующая предельная теорема для $\zeta_{n}(k)$, верная при более широких предположениях, чем теорема 1.

Теорема 2. Пусть выполнено (2). Тогда при $k, n \rightarrow \infty, k \leqslant n$ последовательность случайных величин

$$
\zeta_{n}^{\prime}(k)=\frac{\zeta_{n}(k)-l(k)}{\sqrt{\sigma \ln k}}
$$

слабо сходится $к$ стандартному нормальному закону, где функиия $l(\cdot)$ задана в (3).

Теорема 2 обобщает соответствующие утверждения из [4], [7], [20], [23] и некоторые другие. В свою очередь, теорема 2 выводится из следующей трехмерной тауберовой теоремы.

Теорема 3. Пусть для всех $s, u, v \in(0,1)$ конечна функиия

$$
A(s, u, v)=\sum_{i, j, r \geqslant 0} j^{\beta-1} a(i, j, r) s^{i} u^{j} v^{r}
$$

$(\beta>0, a(i, j, r) \geqslant 0)$, причем для произвольных $\lambda, \mu, \theta>0$ при $n \rightarrow \infty$ для некоторых положительньх последовательностей $k=k(n) \rightarrow \infty u$ $m=m(n) \rightarrow \infty$ имеем

$$
\frac{A\left(e^{-\lambda / k}, e^{-\mu / m}, e^{-\theta / n}\right)}{k m n \varrho(n)} \longrightarrow \lambda^{-1} \mu^{-\beta} \theta^{-\sigma} \Gamma(\beta) \Gamma(\sigma),
$$

где $\sigma>0, \varrho(n)$ - некоторая положительная функиия переменной $n$, $\Gamma(\cdot)$ - гамма-функиия Эйлера. Если $а(i, j, r)$ монотонна по $i$ и по $j u$ nри $n \rightarrow \infty, i \asymp k, j \asymp m, r \geqslant n, r-n=o(n)$ выполнено соотношение

$$
\frac{a(i, j, r)-a(i, j, n)}{a(i, j, r)+a(i, j, n)} \longrightarrow 0
$$

то для произвольных фиксированньц $x, y, z>0$ при $n \rightarrow \infty$

$$
a(x k, y m, z n) \sim \varrho(n) m^{1-\beta} z^{\sigma-1}
$$

$($ при нечельх $s, u, v$ полагаем $a(s, u, v)=a([s],[u],[v]))$. 
3 а м е ч а н и е. Так же как и в теореме 2 из [24], нетрудно заметить, что условие (10) равносильно условию

$$
\frac{a(i, j, r)}{a(i, j, n)} \rightarrow 1
$$

при $n \rightarrow \infty, i \asymp k, j \asymp m, r \geqslant n, r-n=o(n)$. Однако нам будет удобнее проверять это тауберово условие именно в форме (10).

Доказательства теорем 1-3 мы будем проводить в обратном порядке. Сначала мы докажем теорему 3 , затем выведем теорему 2 из теоремы 3 и, наконец, докажем теорему 1 . Зафиксируем $w \geqslant 0$. Положим

$$
a(i, j, r)=p(r) \mathbf{E} \exp \left(\frac{\zeta_{r}(i) w}{\sqrt{l(j)}}\right)
$$

где $i, j, r \in \mathbf{N} \cup\{0\}, p(r)$ определена в $(2), l(j)$ определена в $(3), \zeta_{r}(i)$ определена в (5). (Считаем, что $a(i, j, r)=0$ при $l(j)=0$.) Далее, положим

$$
\varrho(n)=p(n) \exp \left(w \sqrt{l(k)}+\frac{w^{2}}{2}\right) m^{\beta-1}
$$

(как и в теореме 3 , считаем, что $k$ и $m$ суть функции от $n$ ). Сформулируем два вспомогательных утверждения.

Лемма 1. Пусть $k \leqslant n u m \asymp k$ при $n \rightarrow \infty$. Если последовательности $а(i, j, r)$ и $(n)$ задань соотношениями (12) и (13) соответственно, то для некоторого $\beta>1$ и функиии $A(s, u, v)$, определенной равенством (8), справедливо соотношение (9).

Лемма 2. Пусть $k \leqslant n u m \asymp k n р u n \rightarrow \infty$. Тогда для последовательности $a(i, j, r)$, определенной в (12), справедливо соотношение (10).

Д ок аз ат ел ь с т в о ле м м ы 1 . Согласно (8) и (12), при $s=$ $\exp (-\lambda / k), u=\exp (-\mu / m), v=\exp (-\theta / n)$ выполнены соотношения

$$
\begin{aligned}
A(s, u, v) & =\sum_{i, j, r \geqslant 0} j^{\beta-1} a(i, j, r) s^{i} u^{j} v^{r} \\
& =\sum_{i, j \geqslant 0} j^{\beta-1} s^{i} u^{j} \sum_{r \geqslant 0} v^{r} p(r) \mathbf{E} \exp \left(\frac{\zeta_{r}(i) w}{\sqrt{l(j)}}\right) \\
& =\sum_{i, j \geqslant 0} j^{\beta-1} s^{i} u^{j} \exp \left(f(v)+(h(j)-1) \sum_{t \in A(i)} \frac{v^{t}}{t}\right),
\end{aligned}
$$

где

$$
f(v)=\sum_{t \in A} \frac{v^{t}}{t}, \quad h(j)=\exp \left(\frac{w}{\sqrt{l(j)}}\right)
$$


(последнее равенство в (14) справедливо в силу формулы (0.14) из книги $[20$, раздел 3.1$])$. Согласно соотношению 6.3 из работы [1],

$$
\exp (f(v))=\sum_{r \geqslant 0} p(r) v^{r}
$$

Поэтому по абелевой теореме Караматы в силу (2) при $n \rightarrow \infty$

$$
\exp (f(v)) \sim n p(n) \theta^{-\sigma} \Gamma(\sigma)
$$

Из (14) и (17) следует, что при $n \rightarrow \infty$

$$
A(s, u, v) \sim n p(n) \theta^{-\sigma} \Gamma(\sigma) \sum_{i, j \geqslant 0} j^{\beta-1} s^{i} u^{j} \exp \left((h(j)-1) \sum_{t \in A(i)} \frac{v^{t}}{t}\right) .
$$

Поэтому в силу (13) для доказательства леммы 1 нам достаточно показать, что при $n \rightarrow \infty$

$$
\begin{aligned}
\Sigma & \equiv \sum_{i, j \geqslant 0} j^{\beta-1} s^{i} u^{j} \exp \left((h(j)-1) \sum_{t \in A(i)} \frac{v^{t}}{t}\right) \\
& \sim k m^{\beta} \lambda^{-1} \mu^{-\beta} \exp \left(w \sqrt{l(k)}+\frac{w^{2}}{2}\right) \Gamma(\beta) .
\end{aligned}
$$

При $1 \leqslant j \leqslant i$ выполнено следующее неравенство (см. [23, гл. 3, доказательство леммы 3.2 .1$])$ :

$$
l(i)(h(j)-1) \leqslant w \sqrt{l(j)}+C+\alpha \ln \frac{i}{j}
$$

для некоторой постоянной $C>0$, зависящей от $w$, и $\alpha=w+C, \beta=\alpha+1$. Поэтому

$$
\begin{aligned}
\Sigma_{1} & \equiv \sum_{0 \leqslant j \leqslant i / \ln i} j^{\beta-1} s^{i} u^{j} \exp \left((h(j)-1) \sum_{t \in A(i)} \frac{v^{t}}{t}\right) \\
& \leqslant \sum_{0 \leqslant j \leqslant i / \ln i} j^{\beta-1} s^{i} u^{j} \exp ((h(j)-1) l(i)) \\
& =O(1) \sum_{0 \leqslant j \leqslant i / \ln i} i^{\beta-1} s^{i} u^{j} \exp (w \sqrt{l(j)}) \\
& =O(1) \sum_{i \geqslant 0} i^{\beta-1} s^{i} \sum_{0 \leqslant j \leqslant i / \ln i} u^{j} \exp (w \sqrt{l(j)}) \\
& =O(1) \sum_{i \geqslant 0} i^{\beta-1} s^{i} \sum_{0 \leqslant j \leqslant i / \ln i} \exp (w \sqrt{l(j)}) \\
& =O(1) \sum_{i \geqslant 0} i^{\beta-1} s^{i} o(i \exp (w \sqrt{l(i)}))
\end{aligned}
$$




$$
\begin{aligned}
& =o(1) \sum_{i \geqslant 0} i^{\beta} s^{i} \exp (w \sqrt{l(i)}) \\
& =o(1) O\left(k^{\beta+1} \exp (w \sqrt{l(k)})\right) \\
& =o\left(k m^{\beta} \exp (w \sqrt{l(k)})\right)
\end{aligned}
$$

при $n \rightarrow \infty$. Далее,

$$
\begin{aligned}
\Sigma_{2} & \equiv \sum_{i \geqslant 0, j \geqslant i \ln i} j^{\beta-1} s^{i} u^{j} \exp \left((h(j)-1) \sum_{t \in A(i)} \frac{v^{t}}{t}\right) \\
& \leqslant \sum_{i \geqslant 0, j \geqslant i \ln i} j^{\beta-1} s^{i} u^{j} \exp ((h(j)-1) l(i)) .
\end{aligned}
$$

Мы имеем при $j \geqslant i$ :

$$
l(i) h(j) \leqslant l(j) \exp \left(\frac{w}{\sqrt{l(j)}}\right)=l(j)+w \sqrt{l(j)}+O(1)
$$

при $j \rightarrow \infty$. Поэтому для некоторых постоянных $M$ и $c_{1}$ при $j \geqslant M, i \leqslant j$

$$
l(i) h(j) \leqslant l(j)+w \sqrt{l(j)}+c_{1} .
$$

Стало быть, при $j \geqslant i$ для некоторой постоянной $c_{2} \geqslant c_{1}$

$$
l(i)(h(j)-1) \leqslant l(j)-l(i)+w \sqrt{l(j)}+c_{2} \leqslant \ln \frac{j}{i}+w \sqrt{l(j)}+c_{2} .
$$

Из (21) и (22) следует, что при $c_{3}=\exp \left(c_{2}\right)$

$$
\begin{aligned}
\Sigma_{2} & \leqslant c_{3} \sum_{i \geqslant 0} i^{\beta-1} s^{i} \sum_{j \geqslant i \ln i} u^{j} \exp (w \sqrt{l(j)}) \\
& =c_{3} \sum_{i \geqslant 0} i^{\beta-1} s^{i} \sum_{j \geqslant i \ln i} \exp \left(-\frac{\mu j}{2 m}\right) \exp \left(-\frac{\mu j}{2 m}\right) \exp (w \sqrt{l(j)}) \\
& \leqslant c_{3} \sum_{i \geqslant 0} i^{\beta-1} s^{i} \exp \left(-\frac{\mu i \ln i}{2 m}\right) \sum_{j \geqslant 0} \exp \left(-\frac{\mu j}{2 m}\right) \exp (w \sqrt{l(j)}) \\
& =O(m \exp (w \sqrt{l(m)})) \sum_{i \geqslant 0} i^{\beta-1} s^{i} \exp \left(-\frac{\mu i \ln i}{2 m}\right) .
\end{aligned}
$$

Заметим, что для произвольного $\varepsilon>0$

$$
\begin{aligned}
& \sum_{i \geqslant 0} i^{\beta-1} s^{i} \exp \left(-\frac{\mu i \ln i}{2 m}\right) \\
& \leqslant \sum_{i=0}^{[k \varepsilon]} i^{\beta-1}+\sum_{i=[k \varepsilon]+1}^{\infty} i^{\beta-1} s^{i} \exp \left(-\frac{\mu i \ln i}{2 m}\right) \\
& \leqslant c_{4} \varepsilon^{\beta} k^{\beta}+\exp \left(-\frac{\mu \varepsilon k \ln (\varepsilon k)}{2 m}\right) \sum_{i=0}^{\infty} i^{\beta-1} s^{i} \leqslant c_{5} \varepsilon^{\beta} k^{\beta}
\end{aligned}
$$


при достаточно больших $k \geqslant k_{0}$ для некоторых постоянных $c_{4}$ и $c_{5}$ (последнее неравенство выполнено, поскольку $\mu \varepsilon k \ln (\varepsilon k) /(2 m) \rightarrow+\infty$ при $n \rightarrow \infty)$. В силу произвольности $\varepsilon$ отсюда следует, что при $n \rightarrow \infty$

$$
\sum_{i \geqslant 0} i^{\beta-1} s^{i} \exp \left(-\frac{\mu i \ln i}{2 m}\right)=o\left(k^{\beta}\right) .
$$

Из (23) и (24) получаем, что

$$
\Sigma_{2}=o\left(k^{\beta} m \exp (w \sqrt{l(m)})\right)=o\left(k m^{\beta} \exp (w \sqrt{l(k)})\right)
$$

(так как $k \asymp m$, то $\sqrt{l(m)}=\sqrt{l(k)}+o(1))$. Положим

$$
\Sigma_{3} \equiv \sum_{i=0}^{\infty} \sum_{i / \ln i<j<i \ln i} j^{\beta-1} s^{i} u^{j} \exp \left((h(j)-1) \sum_{t \in A(i)} \frac{v^{t}}{t}\right) .
$$

Зафиксируем произвольное $M>0$. Мы имеем при $i \leqslant M n$ :

$$
0 \leqslant \sum_{t \in A(i)} \frac{1-v^{t}}{t}=\sum_{t \in A(i)} \frac{1-\exp (t \theta / n)}{t} \leqslant \sum_{t \in A(i)} \frac{\theta}{n} \leqslant \frac{i \theta}{n} \leqslant M \theta .
$$

Таким образом, при $i \leqslant M n$

$$
\sum_{t \in A(i)} \frac{v^{t}}{t}=l(i)+O(1) \quad(n \rightarrow \infty)
$$

где постоянная в «O» не зависит от $i$. Поэтому

$$
\begin{aligned}
\Sigma_{3}^{\prime} & \equiv \sum_{i=0}^{M n} \sum_{i / \ln } j_{i<j<i \ln i} j^{\beta-1} s^{i} u^{j} \exp \left((h(j)-1) \sum_{t \in A(i)} \frac{v^{t}}{t}\right) \\
& \sim \sum_{i=0}^{M n} \sum_{i / \ln i<j<i \ln i} j^{\beta-1} s^{i} u^{j} \exp ((h(j)-1) l(i)) \quad(n \rightarrow \infty) .
\end{aligned}
$$

При $i / \ln i<j<i \ln i$

$$
\begin{aligned}
(h(j)-1) l(i) & =l(i)\left(\frac{w}{\sqrt{l(j)}}+\frac{w^{2}}{2 l(j)}+O\left((l(j))^{-3 / 2}\right)\right) \\
& =w \sqrt{l(i)}+\frac{w^{2}}{2}+\delta(i, j),
\end{aligned}
$$

где $\delta(i, j) \rightarrow 0$ при $i \rightarrow \infty$ равномерно по $j \in(i / \ln i, i \ln i)$, так как в силу оценки сразу после формулы (9) книги [23, раздел 3.2$]$

$$
\sqrt{l(i)}-\sqrt{l(j)} \longrightarrow 0
$$


при $i \rightarrow \infty$ равномерно по $j \in(i / \ln i, i \ln i)$. Из (27) и (28) следует, что при $n \rightarrow \infty$

$$
\begin{aligned}
\Sigma_{3}^{\prime} & \sim \exp \left(\frac{w^{2}}{2}\right) \sum_{i=0}^{M n} \sum_{i / \ln i<j<i \ln i} j^{\beta-1} s^{i} u^{j} \exp (w \sqrt{l(i)}) \\
& =\exp \left(\frac{w^{2}}{2}\right) \sum_{i=0}^{M n} s^{i} \exp (w \sqrt{l(i)}) \sum_{i / \ln i<j<i \ln i} j^{\beta-1} u^{j} .
\end{aligned}
$$

Мы имеем:

$$
\sum_{0 \leqslant j \leqslant i / \ln i} j^{\beta-1} u^{j} \leqslant \sum_{0 \leqslant j \leqslant i / \ln i} j^{\beta-1}=o\left(i^{\beta}\right) \quad(i \rightarrow \infty) .
$$

Поэтому при $n \rightarrow \infty$

$$
\begin{aligned}
& \sum_{i=0}^{M n} s^{i} \exp (w \sqrt{l(i)}) \sum_{0 \leqslant j \leqslant i / \ln i} j^{\beta-1} u^{j} \\
& =o\left(\sum_{i=0}^{\infty} i^{\beta} s^{i} \exp (w \sqrt{l(i)})\right)=o\left(k^{\beta+1} \exp (w \sqrt{l(k)})\right) .
\end{aligned}
$$

Точно так же, как и в оценках (23)-(25), получаем, что

$$
\sum_{i=0}^{M n} s^{i} \exp (w \sqrt{l(i)}) \sum_{j \geqslant i \ln i} j^{\beta-1} u^{j}=o\left(k^{\beta+1} \exp (w \sqrt{l(k)})\right)
$$

при $n \rightarrow \infty$. Поэтому

$$
\begin{aligned}
\sum_{i=0}^{M n} s^{i} & \exp (w \sqrt{l(i)}) \sum_{i / \ln i<j<i \ln i} j^{\beta-1} u^{j} \\
= & \sum_{i=0}^{M n} s^{i} \exp (w \sqrt{l(i)}) \sum_{j=0}^{\infty} j^{\beta-1} u^{j}+o\left(k^{\beta+1} \exp (w \sqrt{l(k)})\right)
\end{aligned}
$$

при $n \rightarrow \infty$. Но

$$
\sum_{j=0}^{\infty} j^{\beta-1} u^{j}=\sum_{j=0}^{\infty} j^{\beta-1} \exp \left(-\frac{\mu j}{m}\right) \sim \Gamma(\beta) m^{\beta} \mu^{-\beta} \quad(n \rightarrow \infty) .
$$

Из (29), (30) и (31) следует, что при $n \rightarrow \infty$

$$
\begin{aligned}
\Sigma_{3}^{\prime}= & (1+o(1)) \exp \left(\frac{w^{2}}{2}\right) \sum_{i=0}^{M n} s^{i} \exp (w \sqrt{l(i)}) \sum_{j=0}^{\infty} j^{\beta-1} u^{j} \\
& +o\left(k^{\beta+1} \exp (w \sqrt{l(k)})\right) \\
= & (1+o(1)) \Gamma(\beta)\left(\frac{m}{\mu}\right)^{\beta} \exp \left(\frac{w^{2}}{2}\right) \sum_{i=0}^{M n} s^{i} \exp (w \sqrt{l(i)}) \\
& +o\left(k^{\beta+1} \exp (w \sqrt{l(k)})\right) .
\end{aligned}
$$


Далее,

$$
\begin{aligned}
\Sigma_{3}^{\prime \prime} & \equiv \sum_{i=M n+1}^{\infty} \sum_{i / \ln i<j<i \ln i} j^{\beta-1} s^{i} u^{j} \exp \left((h(j)-1) \sum_{t \in A(i)} \frac{v^{t}}{t}\right) \\
& \leqslant \sum_{i=M n+1}^{\infty} \sum_{i / \ln i<j<i \ln i} j^{\beta-1} s^{i} u^{j} \exp ((h(j)-1) l(i)) .
\end{aligned}
$$

Оценивая $\Sigma_{3}^{\prime \prime}$ точно так же, как и $\Sigma_{3}^{\prime}$, получаем, что для некоторой постоянной $c_{6}$, не зависящей от $M$,

$$
\Sigma_{3}^{\prime \prime} \leqslant c_{6} m^{\beta} \sum_{i=M n+1}^{\infty} s^{i} \exp (w \sqrt{l(i)})+\varepsilon(k) k^{\beta+1} \exp (w \sqrt{l(k)}),
$$

где $\varepsilon(k) \rightarrow 0$ при $k \rightarrow \infty$. Мы имеем:

$$
\begin{aligned}
& \sum_{i=M n+1}^{\infty} s^{i} \exp (w \sqrt{l(i)})=\sum_{i=M n+1}^{\infty} \exp \left(-\frac{\lambda i}{k}\right) \exp (w \sqrt{l(i)}) \\
& \quad \leqslant \exp \left(-\frac{\lambda M}{2}\right) \sum_{i=M n+1}^{\infty} \exp \left(-\frac{\lambda i}{2 k}\right) \exp (w \sqrt{l(i)}) \\
& \quad=(1+o(1)) \exp \left(-\frac{\lambda M}{2}\right) \frac{2 k}{\lambda} \exp (w \sqrt{l(k)}) \quad(n \rightarrow \infty) .
\end{aligned}
$$

Из (33) и (34) следует, что

$$
\limsup _{n \rightarrow \infty} \frac{\Sigma_{3}^{\prime \prime}}{k m^{\beta} \exp (w \sqrt{l(k)})} \leqslant 2 c_{6} \frac{\exp (-\lambda M / 2)}{\lambda} .
$$

Далее,

$$
\limsup _{n \rightarrow \infty} \frac{\sum_{i=0}^{M n} s^{i} \exp (w \sqrt{l(i)})}{k \exp (w \sqrt{l(k)})} \leqslant \lim _{n \rightarrow \infty} \frac{\sum_{i=0}^{\infty} s^{i} \exp (w \sqrt{l(i)})}{k \exp (w \sqrt{l(k)})}=\frac{1}{\lambda} .
$$

Из (34) следует, что

$$
\begin{aligned}
\liminf _{n \rightarrow \infty} & \frac{\sum_{i=0}^{M n} s^{i} \exp (w \sqrt{l(i)})}{k \exp (w \sqrt{l(k)})} \geqslant \lim _{n \rightarrow \infty} \frac{\sum_{i=0}^{\infty} s^{i} \exp (w \sqrt{l(i)})}{k \exp (w \sqrt{l(k)})} \\
& -\limsup _{n \rightarrow \infty} \frac{\sum_{i=M n}^{\infty} s^{i} \exp (w \sqrt{l(i)})}{k \exp (w \sqrt{l(k)})} \geqslant \frac{1}{\lambda}-\frac{2}{\lambda} \exp \left(-\frac{\lambda M}{2}\right) .
\end{aligned}
$$

Из (32) и (37) получаем, что

$$
\begin{gathered}
\liminf _{n \rightarrow \infty} \frac{\Sigma_{3}}{k m^{\beta} \exp (w \sqrt{l(k)})} \geqslant \liminf _{n \rightarrow \infty} \frac{\Sigma_{3}^{\prime}}{k m^{\beta} \exp (w \sqrt{l(k)})} \\
\geqslant \frac{\Gamma(\beta)}{\mu^{\beta}} \exp \left(\frac{w^{2}}{2}\right)\left(\frac{1}{\lambda}-\frac{2}{\lambda} \exp \left(-\frac{\lambda M}{2}\right)\right) .
\end{gathered}
$$


Из (32), (35) и (36) следует, что

$$
\begin{aligned}
& \limsup _{n \rightarrow \infty} \frac{\Sigma_{3}}{k m^{\beta} \exp (w \sqrt{l(k)})} \\
& \quad \leqslant \limsup _{n \rightarrow \infty} \frac{\Sigma_{3}^{\prime}}{k m^{\beta} \exp (w \sqrt{l(k)})}+\limsup _{n \rightarrow \infty} \frac{\Sigma_{3}^{\prime \prime}}{k m^{\beta} \exp (w \sqrt{l(k)})} \\
& \quad \leqslant \frac{\Gamma(\beta)}{\mu^{\beta}} \exp \left(\frac{w^{2}}{2}\right) \frac{1}{\lambda}+2 c_{6} \frac{\exp (-\lambda M / 2)}{\lambda} .
\end{aligned}
$$

Так как $\Sigma_{3}$ не зависит от $M$, то, устремляя в (38) и (39) $M$ к бесконечности, убеждаемся в том, что на самом деле существует

$$
\lim _{n \rightarrow \infty} \frac{\Sigma_{3}}{k m^{\beta} \exp (w \sqrt{l(k)})}=\frac{\Gamma(\beta)}{\mu^{\beta}} \exp \left(\frac{w^{2}}{2}\right) \frac{1}{\lambda} .
$$

С учетом оценок $(20)$ и $(25)$ для $\Sigma_{1}$ и $\Sigma_{2}$, из последнего соотношения вытекает (18). Лемма 1 доказана.

Д о к а 3 а т е л ь с т в о л е м м ы 2. Доказательство леммы 2 похоже на доказательство леммы 1 работы [24], поэтому мы отметим только места, в которых имеются отличия. В доказательстве леммы 2 константы $c, c_{1}, c_{2}, \ldots$, вообще говоря, не совпадают с константами $c, c_{1}, c_{2}, \ldots$ доказательства леммы 1 . Мы имеем:

$$
a(i, j, n)=p(n)+\sum_{\tau=1}^{n} \frac{(h(j)-1)^{\tau}}{\tau !} S_{\tau}(i, n)=p(n)+\sum_{\tau=1}^{n} b_{\tau}(i, j, n),
$$

где

$$
S_{\tau}(i, n)=\sum_{i_{1}, \ldots, i_{\tau} \in A(i)} \frac{1}{i_{1} \cdots i_{\tau}} p\left(n-i_{1}-\cdots-i_{\tau}\right)
$$

(см. доказательство леммы 1 из [24]). Для данной последовательности $r=r(n), r=n+o(n)$ найдстся последовательность $g(n)>0, g(n)=o(n)$, $(r-n) / g(n) \rightarrow 0$. Для

$$
I_{1}(\tau, i, n)=\sum_{i_{1}, \ldots, i_{\tau} \in A(i), \sum_{l=1}^{\tau} i_{l} \in[n-g(n), n]} \frac{1}{i_{1} \cdots i_{\tau}} p\left(n-i_{1}-\cdots-i_{\tau}\right)
$$

справедлива оценка:

$$
I_{1}(\tau, i, n) \leqslant \delta(n) \tau^{2} p(n)(l(i))^{\tau-1},
$$

где $\delta(n) \rightarrow 0$ при $n \rightarrow \infty$ (см. соотношения (23)-(27) из [24]). Зафиксируем $c \in(0,1)$ и положим

$$
\begin{aligned}
I_{2}(\tau, i, n) & =\sum_{i_{1}, \ldots, i_{\tau} \in A(i), \sum_{l=1}^{\tau} i_{l} \in(c n, n]} \frac{1}{i_{1} \cdots i_{\tau}} p\left(n-i_{1}-\cdots-i_{\tau}\right), \\
I_{3}(\tau, i, n) & =\sum_{i_{1}, \ldots, i_{\tau} \in A(i), \sum_{l=1}^{\tau} i_{l} \leqslant c n} \frac{1}{i_{1} \cdots i_{\tau}} p\left(n-i_{1}-\cdots-i_{\tau}\right) .
\end{aligned}
$$


Для $I_{2}(\tau, i, n)$ и $I_{3}(\tau, i, n)$ мы имеем следующие оценки:

$$
\begin{aligned}
& I_{2}(\tau, i, n)=O\left(\tau^{2} p(n)(l(i))^{\tau-1}\right), \\
& I_{3}(\tau, i, n)=O\left(p(n)(l(i))^{\tau}\right),
\end{aligned}
$$

где константы в «O» не зависят от $\tau$ и $i$. Далее, оценим снизу величину $S_{\tau}(i, n)$ при $\tau \leqslant c n:$

$$
\begin{aligned}
S_{\tau}(i, n) & \geqslant \sum_{i_{1}, \ldots, i_{\tau} \in A(i), \sum_{l=1}^{\tau} i_{l} \leqslant c n} \frac{1}{i_{1} \cdots i_{\tau}} p\left(n-i_{1}-\cdots-i_{\tau}\right) \\
& \geqslant c_{1} p(n) \sum_{i_{1}, \ldots, i_{\tau} \in A(i), \sum_{l=1}^{\tau} i_{l} \leqslant c n} \frac{1}{i_{1} \cdots i_{\tau}} \\
& \geqslant c_{1} p(n) \sum_{i_{1}, \ldots, i_{\tau} \in A(t)} \frac{1}{i_{1} \cdots i_{\tau}}=c_{1} p(n)(l(t))^{\tau}
\end{aligned}
$$

где $t=\min (c n / \tau, i)$. Если $i \leqslant c n / \tau$, то

$$
S_{\tau}(i, n) \geqslant c_{1} p(n)(l(i))^{\tau} .
$$

Пусть $i>c n / \tau$. Зафиксируем произвольные $u_{1}$ и $u_{2}, 0<u_{1}<u_{2}<\infty$. Будем считать далее, что $i \in\left[u_{1} k, u_{2} k\right]$. Тогда так как $i \leqslant u_{2} k \leqslant u_{2} n$, то при $c_{2}=u_{2} c$

$$
l(i)-l\left(\frac{c n}{\tau}\right) \geqslant l(i)-l\left(\frac{c_{2} i}{\tau}\right) .
$$

Далее точно так же, как и в [24], получаем, что для некоторой постоянной $M>0$ существуют такие постоянные $c_{3} \in(0, \infty)$ и $n_{1} \in \mathbf{N}$, что при $n \geqslant n_{1}, \tau \leqslant M \sqrt{\ln k}$ и $i \in\left[u_{1} k, u_{2} k\right]$

$$
(l(t))^{\tau} \geqslant\left(l\left(\frac{c_{2} i}{\tau}\right)\right)^{\tau} \geqslant c_{3}(l(i))^{\tau}
$$

Поэтому для некоторой постоянной $c_{4} \in(0, \infty)$ при $n \geqslant n_{1}, \tau \leqslant M \sqrt{\ln k}$ и $i \in\left[u_{1} k, u_{2} k\right]$

$$
S_{\tau}(i, n) \geqslant c_{4} p(n)(l(i))^{\tau} .
$$

Из (44)-(47) получаем, что при $n \rightarrow \infty$ и $\tau \leqslant n$

$$
S_{\tau}(i, n)=I_{2}(\tau, i, n)+I_{3}(\tau, i, n)=O\left(\tau^{2} p(n)(l(i))^{\tau}\right) .
$$

Значит, существуют такие $n_{2}>n_{1}$ и $c_{5} \in(0, \infty)$, что при $n \geqslant n_{2}, \tau \leqslant n$ и $i \in\left[u_{1} k, u_{2} k\right]$

$$
S_{\tau}(i, n) \leqslant c_{5} \tau^{2} p(n)(1+\ln i)^{\tau} \leqslant c_{5} \tau^{2} p(n)(2 \ln k)^{\tau} .
$$


Далее, при $n \rightarrow \infty$ и $j \asymp m \asymp k$

$$
h(j)-1=(1+o(1)) \frac{w}{\sqrt{l(j)}}=(1+o(1)) \frac{w}{\sqrt{\sigma \ln j}}=(1+o(1)) \frac{w}{\sqrt{\sigma} \sqrt{\ln k}} .
$$

Стало быть, для произвольных постоянных $s_{1}$ и $s_{2}, 0<s_{1}<s_{2}<\infty$, и некоторой постоянной $c_{6} \in(w / \sqrt{\sigma}, \infty)$, зависящей от $s_{1}$ и $s_{2}$, существует такое $n_{3}>n_{2}$, что при $n \geqslant n_{3}$ и $j \in\left[s_{1} k, s_{2} k\right]$

$$
h(j)-1 \leqslant \frac{c_{6}}{\sqrt{\ln k}} .
$$

В силу (49) и (50), при $n \geqslant n_{3}, i \in\left[u_{1} k, u_{2} k\right]$ и $j \in\left[s_{1} k, s_{2} k\right]$

$$
\begin{aligned}
b_{\tau}(i, j, n) & =\frac{(h(j)-1)^{\tau}}{\tau !} S_{\tau}(i, n) \leqslant c_{5} \tau^{2} c_{6}^{\tau} \frac{(2 \ln k)^{\tau}}{\tau !(\ln k)^{\tau / 2}} p(n) \\
& =c_{5} \tau^{2} c_{7}^{\tau} \frac{(\sqrt{\ln k})^{\tau}}{\tau !} p(n),
\end{aligned}
$$

где $c_{7}=2 c_{6}$. Используя для $\tau$ ! формулу Стирлинга, убеждаемся в том, что при $i \in\left[u_{1} k, u_{2} k\right]$ и $j \in\left[s_{1} k, s_{2} k\right]$

$$
\sum_{\tau>e^{2} c_{7} \sqrt{\ln k}} b_{\tau}(i, j, n)=o(p(n)) \quad(n \rightarrow \infty) .
$$

Дальнейшее доказательство леммы 2 с учетом полученных оценок практически дословно повторяет доказательство леммы 1 из [24].

Д ок аз а т е л ь с т в о т е о р е м ы 3 . Пусть $\mathfrak{B}-$ совокупность ограниченных борелевских множеств из $\mathbf{R}_{+}^{3}=\left\{\mathbf{x}=\left(x_{1}, x_{2}, x_{3}\right) \in \mathbf{R}^{3}\right.$ : $\left.x_{1}, x_{2}, x_{3} \geqslant 0\right\}$. Для произвольного множества $B \in \mathfrak{B}$ положим

$$
\mu_{n}(B)=\sum_{i, j, r:(i / k, j / m, r / n) \in B} \frac{j^{\beta-1} a(i, j, r)}{k m n \varrho(n)} .
$$

В силу (9) для преобразования Лапласа мер $\mu_{n}$ выполнены соотношения

$$
\begin{aligned}
\widetilde{\mu}_{n}(\mathbf{y}) & \equiv \int_{\mathbf{R}_{+}^{3}} e^{-(\mathbf{x}, \mathbf{y})} \mu_{n}(d \mathbf{x})=\frac{A\left(e^{-y_{1} k}, e^{-y_{2} m}, e^{-y_{3} n}\right)}{k m n \varrho(n)} \\
& \longrightarrow \psi(\mathbf{y})=\widetilde{\mu}(\mathbf{y})=\int_{\mathbf{R}_{+}^{3}} e^{-(\mathbf{x}, \mathbf{y})} x_{2}^{\beta-1} x_{3}^{\sigma-1} d x_{1} d x_{2} d x_{3}
\end{aligned}
$$

при $n \rightarrow \infty$ для произвольного фиксированного $\mathbf{y} \in \operatorname{Int} \mathbf{R}_{+}^{3}$. Таким образом, предельная функция $\psi(\mathbf{y})$ в (53) является преобразованием Лапласа абсолютно непрерывной меры $\mu$ :

$$
\mu(B)=\int_{B} e^{-(\mathbf{x}, \mathbf{y})} x_{2}^{\beta-1} x_{3}^{\sigma-1} d x_{1} d x_{2} d x_{3}, \quad B \in \mathfrak{B} .
$$


Из (53) в силу тауберовой теоремы 1.3 .2 из [23] следует, что при $n \rightarrow \infty$

$$
\mu_{n} \Rightarrow \mu
$$

т.е. для произвольного $B \in \mathfrak{B}$ такого, что $\mu(\partial B)=0$, мы имеем:

$$
\mu_{n}(B) \longrightarrow \mu(B) \quad(n \rightarrow \infty)
$$

Предположим для определенности, что $a(i, j, r)$ не убывает по $i$ и не возрастает по $j$ и $\beta>1$, как в нашем конкретном приложении тауберовой теоремы 3. Из дальнейшего будет ясно, как поступать в других случаях. Зафиксируем произвольные $x, y, z>0$. Для произвольных $\delta_{1}, \delta_{2}, \delta_{3} \in$ $(0,1)$, используя (54) и (55) при $B=\left\{\left(x_{1}, x_{2}, x_{3}\right): x \leqslant x_{1} \leqslant x\left(1+\delta_{1}\right)\right.$, $\left.y\left(1-\delta_{2}\right) \leqslant x_{2} \leqslant y, z \leqslant x_{3} \leqslant z\left(1+\delta_{3}\right)\right\}$, мы имеем:

$$
\Sigma \equiv \sum^{*} \frac{j^{\beta-1} a(i, j, r)}{k m n \varrho(n)} \longrightarrow \delta_{1} x \frac{y^{\beta}\left(1-\left(1-\delta_{2}\right)^{\beta}\right)}{\beta} \frac{z^{\sigma}\left(\left(1+\delta_{3}\right)^{\sigma}-1\right)}{\sigma}
$$

при $n \rightarrow \infty$, где $\sum^{*}$ означает суммирование по $i \in\left[k x, k x\left(1+\delta_{1}\right)\right], j \in$ $\left[m y\left(1-\delta_{2}\right), m y\right], r \in\left[n z, n z\left(1+\delta_{3}\right)\right]$. Далее,

$$
\Sigma \geqslant \sum^{*} \frac{\left(m y\left(1-\delta_{2}\right)\right)^{\beta-1} a(k x, m y, r)}{k m n \varrho(n)} .
$$

Согласно (10) и замечанию после теоремы 3 , для произвольного $\varepsilon \in(0,1)$ найдутся такие $\delta_{3}$ и $n_{0}$, что при $n \geqslant n_{0}$ и $|r-[n z]| \leqslant \delta_{3} n z$ выполнено неравенство

$$
|a(k x, m y, r)-a(k x, m y, n z)| \leqslant \varepsilon a(k x, m y, n z) .
$$

Стало быть, при $n \geqslant n_{0}$ мы получаем из (57) и (58):

$$
\Sigma \geqslant \frac{(1-\varepsilon) a(k x, m y, n z)\left(y\left(1-\delta_{2}\right)\right)^{\beta-1}}{m^{1-\beta} \varrho(n)} \frac{\sum^{*} 1}{m n k} .
$$

Из (56) и (59) следует, что

$$
\begin{aligned}
\limsup _{n \rightarrow \infty} \frac{a(k x, m y, n z)}{m^{1-\beta} \varrho(n)} \leqslant \frac{\left(y\left(1-\delta_{2}\right)\right)^{1-\beta}}{1-\varepsilon} \lim _{n \rightarrow \infty} \frac{\sum m n k}{\sum^{*} 1} \\
\quad=\frac{\delta_{1} x\left(y\left(1-\delta_{2}\right)\right)^{1-\beta} y^{\beta}\left(1-\left(1-\delta_{2}\right)^{\beta}\right) z^{\sigma}\left(\left(1+\delta_{3}\right)^{\sigma}-1\right)}{(1-\varepsilon) \delta_{1} x \beta \delta_{2} y \sigma \delta_{3} z} \\
\quad=\frac{1}{1-\varepsilon} \frac{\left(1-\delta_{2}\right)^{1-\beta}\left(1-\left(1-\delta_{2}\right)^{\beta}\right)}{\beta \delta_{2}} z^{\sigma-1} \frac{\left(1+\delta_{3}\right)^{\sigma}-1}{\sigma \delta_{3}} .
\end{aligned}
$$

Поскольку левая часть $(60)$ не зависит от $\varepsilon, \delta_{2}$ и $\delta_{3}$, то, устремляя $\varepsilon, \delta_{2}$ и $\delta_{3}$ к нулю в правой части (60), убеждаемся в том, что

$$
\limsup _{n \rightarrow \infty} \frac{a(k x, m y, n z)}{m^{1-\beta} \varrho(n)} \leqslant z^{\sigma-1} .
$$


Далее положим $B^{\prime}=\left\{\left(x_{1}, x_{2}, x_{3}\right): x\left(1-\delta_{1}\right) \leqslant x_{1} \leqslant x, y \leqslant x_{2} \leqslant y(1+\right.$ $\left.\left.\delta_{2}\right), z\left(1-\delta_{3}\right) \leqslant x_{3} \leqslant z\right\}$. Используя (54) и (55) для $B^{\prime}$, мы имеем:

$$
\Sigma^{\prime} \equiv \sum^{* *} \frac{j^{\beta-1} a(i, j, r)}{k m n \varrho(n)} \rightarrow \delta_{1} x \frac{y^{\beta}\left(\left(1+\delta_{2}\right)^{\beta}-1\right)}{\beta} \frac{z^{\sigma}\left(1-\left(1-\delta_{3}\right)^{\sigma}\right)}{\sigma},
$$

где $\sum^{* *}$ означает суммирование по $i \in\left[k x\left(1-\delta_{1}\right), k x\right], j \in\left[m y, m y\left(1+\delta_{2}\right)\right]$, $r \in\left[n z\left(1-\delta_{3}\right), n z\right]$. С учетом (58) мы получаем, что при $n \geqslant n_{0}$

$$
\Sigma^{\prime} \leqslant \frac{(1+\varepsilon) a(k x, m y, n z)\left(y\left(1+\delta_{2}\right)\right)^{\beta-1}}{m^{1-\beta} \varrho(n)} \frac{\sum^{* *} 1}{m n k} .
$$

Из (62) и (63) следует, что

$$
\begin{aligned}
\liminf _{n \rightarrow \infty} \frac{a(k x, m y, n z)}{m^{1-\beta} \varrho(n)} & \geqslant \frac{\left(y\left(1+\delta_{2}\right)\right)^{1-\beta}}{1+\varepsilon} \lim _{n \rightarrow \infty} \frac{\sum^{\prime} m n k}{\sum^{* *} 1} \\
& =\frac{\delta_{1} x\left(y\left(1+\delta_{2}\right)\right)^{1-\beta} y^{\beta}\left(\left(1+\delta_{2}\right)^{\beta}-1\right) z^{\sigma}\left(1-\left(1-\delta_{3}\right)^{\sigma}\right)}{(1+\varepsilon) \delta_{1} x \beta \delta_{2} y \sigma \delta_{3} z} \\
& =\frac{1}{1+\varepsilon} \frac{\left(1+\delta_{2}\right)^{\beta}-1}{\beta \delta_{2}\left(1+\delta_{2}\right)^{\beta-1}} z^{\sigma-1} \frac{\left(1-\left(1-\delta_{3}\right)^{\sigma}\right.}{\sigma \delta_{3}}
\end{aligned}
$$

Поскольку левая часть (64) не зависит от $\varepsilon, \delta_{2}$ и $\delta_{3}$, то, устремляя $\varepsilon, \delta_{2}$ и $\delta_{3}$ к нулю в правой части (64), убеждаемся в том, что

$$
\liminf _{n \rightarrow \infty} \frac{a(k x, m y, n z)}{m^{1-\beta} \varrho(n)} \geqslant z^{\sigma-1} .
$$

Из (61) и (65) следует (11). Теорема 3 доказана.

Д ок аз а т ель с т в о т е о ре мы 2. Из лемм 1 и 2 следует, что для $a(i, j, r)$ и $\varrho(n)$, удовлетворяющих (12) и $(13)$, и некоторого $\beta>1$ выполнены все предположения теоремы 3 . По этой теореме имеем при $m=k \leqslant n, x=y=z=1$ и $k, n \rightarrow \infty$

$$
a(k, k, n) \sim \varrho(n) m^{1-\beta},
$$

откуда, учитывая (12) и (13), получаем, что

$$
\mathbf{E} \exp \left(\frac{\zeta_{n}(k) w}{\sqrt{l(k)}}\right) \sim \exp \left(w \sqrt{l(k)}+\frac{w^{2}}{2}\right) .
$$

Иными словами, при $k, n \rightarrow \infty, k \leqslant n$

$$
\mathbf{E} \exp \left(\left(\frac{\zeta_{n}(k)-l(k)}{\sqrt{l(k)}}\right) w\right) \rightarrow \exp \left(\frac{w^{2}}{2}\right) .
$$

Поэтому при $k, n \rightarrow \infty, k \leqslant n$ последовательность случайных величин

$$
\zeta_{n}^{\prime \prime}(k)=\frac{\zeta_{n}(k)-l(k)}{\sqrt{l(k)}}
$$


слабо сходится к стандартному нормальному закону. Так как $l(k) \sim$ $\sigma \ln k$ при $k \rightarrow \infty$ (см. (4)), то отсюда следует, что и последовательность $\zeta_{n}^{\prime}(k)$ слабо сходится к стандартному нормальному закону. Теорема 2 доказана.

Д ок аз а т ел ь с т в о т е о ре м ы 1 . Зафиксируем произвольное действительное $x$. Положим в равенстве (6)

$$
r=\exp \left(\frac{g(n)}{\sigma}+x \frac{\sqrt{g(n)}}{\sigma}\right), \quad m=g(n)+o(\sqrt{g(n)}) .
$$

Мы имеем:

$$
\begin{aligned}
& \mathbf{P}\{\sigma\left.\ln \eta_{n}(m) \leqslant g(n)+x \sqrt{g(n)}\right\}=\mathbf{P}\left\{\eta_{n}(m) \leqslant r\right\} \\
& \quad=\mathbf{P}\left\{\zeta_{n}(r) \geqslant m\right\}=\mathbf{P}\left\{\zeta_{n}(r) \geqslant l(r)-l(r)+g(n)+o(\sqrt{g(n)})\right\} .
\end{aligned}
$$

Так как $l(r)=\sigma \ln r+o(\sqrt{\ln r})=g(n)+(x+o(1)) \sqrt{g(n)}$ (см. (3)), то

$$
\begin{aligned}
\mathbf{P}\{\sigma & \left.\ln \eta_{n}(m) \leqslant g(n)+x \sqrt{g(n)}\right\} \\
= & \mathbf{P}\left\{\zeta_{n}(r) \geqslant l(r)-g(n)-(x+o(1)) \sqrt{g(n)}+g(n)+o(\sqrt{g(n)})\right\} \\
& =\mathbf{P}\left\{\zeta_{n}(r) \geqslant l(r)-(x+o(1)) \sqrt{g(n)}\right\} .
\end{aligned}
$$

Но так как $\ln r=(1+o(1)) g(n) / \sigma$, то

$$
g(n)=\sigma \ln r(1+o(1)) .
$$

Подставляя (67) в (66), получаем, что

$$
\begin{aligned}
& \mathbf{P}\left\{\sigma \ln \eta_{n}(m) \leqslant g(n)+x \sqrt{g(n)}\right\} \\
& \quad=\mathbf{P}\left\{\zeta_{n}(r) \geqslant l(r)-(x+o(1)) \sqrt{\sigma \ln r}\right\} \longrightarrow 1-\Phi(-x)=\Phi(x)
\end{aligned}
$$

согласно теореме 2 , где $\Phi(\cdot)$ - функция распределения стандартного нормального закона, так как $r \rightarrow \infty, r / n \rightarrow 0$ при $n \rightarrow \infty$. Действительно, поскольку $(\sigma \ln n-g(n)) / \sqrt{\ln n} \longrightarrow+\infty$, то

$$
\frac{g(n)}{\sigma}=\ln n-M(n) \sqrt{\ln n},
$$

где $M(n) \longrightarrow+\infty$ при $n \rightarrow \infty$, откуда

$\frac{r}{n}=\exp \left(\frac{g(n)}{\sigma}-\ln n+x \frac{\sqrt{g(n)}}{\sigma}\right)=\exp (-M(n) \sqrt{\ln n}+O(\sqrt{\ln n})) \longrightarrow 0$ при $n \rightarrow \infty$. Теорема 1 доказана.

В заключение автор выражает глубокую признательность рецензенту за ценные замечания, которые были учтены при подготовке рукописи к печати. 


\section{СПИСОК ЛИТЕРАТУРЫ}

1. Bender E. A. Asymptotic methods in enumeration. - SIAM Rev., 1974, v. 16, № 4, p. 485-515; corrections: ibid., 1976, v. 18, № 2, p. 292.

2. Болотников Ю.В., Сачков В.Н., Тараканов В.Е. Асимптотическая нормальность некоторых величин, связанных с цикловой структурой случайных подстановок. - Матем. сб., 1976, т. 99, № 1, с. 121-133.

3. Вольнеи Л. М. Пример нестандартной асимптотики числа подстановок с ограничениями на длины циклов. - Вероятностные процессы и их приложения. М.: МИЭМ, 1989, с. 85-90.

4. Гончаров В.Л. Из области комбинаторики. - Изв. АН СССР, 1944, т. 8, № 1, c. $3-48$.

5. Ивченко Г.И., Медведев Ю.И. Метод В. Л.Гончарова и его развитие в анализе различных моделей случайных подстановок. - Теория вероятн. и ее примен., 2002 , т. 47 , в. 3 , с. $558-566$.

6. Колчин $B . \Phi$. Одна задача о размещении частиц по ячейкам и циклы случайных подстановок. - Теория вероятн. и ее примен., 1971, т. 16, в. 1, с. 67-82.

7. Колчин В. Ф. Случайные отображения. М.: Наука, 1984, 206 с.

8. Колчин В.Ф. О числе подстановок с ограничениями на длины циклов. - Дискретн. матем., 1989, т. 1, № 2, с. 97-109.

9. Kolchin V.F. The number of permutations with cycle lengths from a fixed set. Random Graphs (Poznań, 1989), v. 2. New York: Wiley, 1992, p. 139-149.

10. Колчин В. Ф. Случайные графы. М.: Физматлит, 2000, 255 с.

11. Минеев М. П., Павлов А. И. О числе подстановок специального вида. - Матем. сб., 1976 , т. 99, № 3, с. 468-476.

12. Минеев М. П., Павлов А.И. Об уравнении в подстановках. — Тр. МИАН, 1976, т. 142 , c. $182-194$.

13. Павлов А.И. О некоторых классах подстановок с теоретико-числовыми ограничениями на длины циклов. - Матем. сб., 1986, т. 129, № 2, с. 252-263.

14. Павлов А.И. О подстановках с длинами циклов из заданного множества. Теория вероятн. и ее примен., 1986, т. 31 , в. 3, с. 618-619.

15. Павлов А. И. О числе подстановок с длинами циклов из заданного множества. Дискретн. матем., 1991, т. 3, № 3, с. 109-123.

16. Павлов А. И. О двух классах подстановок с теоретико-числовыми ограничениями на длины циклов. - Матем. заметки, 1997, т. 62, № 6, с. 881-891.

17. Сачков B. Н. Отображения конечного множества с ограничениями на контуры и высоту. - Теория вероятн. и ее примен., 1972, т. 17, в. 4, с. 679-694.

18. Сачков В. Н. Случайные отображения ограниченной высоты. - Теория вероятн. и ее примен., 1973, т. 18 , в. 1, с. $122-132$.

19. Сачков В. Н. Комбинаторные методы дискретной математики. М.: Наука, 1977, $319 \mathrm{c}$.

20. Сачков В. Н. Вероятностные методы в комбинаторном анализе. М.: Наука, 1978, $287 \mathrm{c.}$

21. Сенета Е. Правильно меняющиеся функции. М.: Наука, 1985, 141 с.

22. Якьлив А.Л. Распределение длины $m$-го максимального цикла случайной $A$-подстановки. - Дискретная матем., 2005, т. 17, № 4, с. 40-58.

23. Якьмив А.Л. Вероятностные приложения тауберовых теорем. М: Физматлит, $2005,256 \mathrm{c}$.

24. Якьмив А.Л. Предельная теорема для общего числа циклов случайной $A$-подстановки. - Теория вероятн. и ее примен., 2007, т. 52 , в. 1, с. $69-83$.

25. Якьлив А.Л. Случайные $A$-подстановки: сходимость к пуассоновскому процессу. - Матем. заметки, 2007, т. 81, № 6, с. 939-947.

Поступила в редакцию

1.XII.2006

Исправленный вариант 31.X.2007 